\section{EMBRYRIDDLE Aeronautical University}

SCHOLARLY COMMONS
International Journal of Aviation, Aeronautics, and Aerospace

\title{
A Comparison of Cervical and Trunk Musculoskeletal Characteristics between Female and Male Army Helicopter Pilots
}

\author{
Takashi Nagai \\ University of Pittsburgh, tnagai@pitt.edu \\ Karen A. Keenan \\ University of Pittsburgh, kak170@pitt.edu \\ John P. Abt \\ University of Kentucky, john.abt@uky.edu \\ Timothy C. Sell \\ Duke University, timothy.sell@duke.edu \\ Brian W. Smalley \\ US Army Aeromedical Activity, brian.w.smalley.civ@mail.mil \\ Michael D. Wirt \\ US Army Institute of Surgical Research, michael.d.wirt.mil@mail.mil \\ Scott M. Lephart \\ University of Kentucky, scott.lephart@uky.edu
}

Follow this and additional works at: https://commons.erau.edu/ijaaa

Part of the Biomechanics Commons, Exercise Physiology Commons, Motor Control Commons, Musculoskeletal Diseases Commons, Orthopedics Commons, Preventive Medicine Commons, and the Sports Medicine Commons

\section{Scholarly Commons Citation}

Nagai, T., Keenan, K. A., Abt, J. P., Sell, T. C., Smalley, B. W., Wirt, M. D., \& Lephart, S. M. (2016). A Comparison of Cervical and Trunk Musculoskeletal Characteristics between Female and Male Army Helicopter Pilots. International Journal of Aviation, Aeronautics, and Aerospace, 3(3). https://doi.org/ 10.15394/ijaaa.2016.1136

This Article is brought to you for free and open access by the Journals at Scholarly Commons. It has been accepted for inclusion in International Journal of Aviation, Aeronautics, and Aerospace by an authorized administrator of Scholarly Commons. For more information, please contact commons@erau.edu. 


\section{A Comparison of Cervical and Trunk Musculoskeletal Characteristics between Female and Male Army Helicopter Pilots}

\section{Cover Page Footnote}

This study was supported by the U.S. Army Medical Research and Materiel Command under Award No. W81XWH-11-2-0097. Opinions, interpretations, conclusions, and recommendations are those of the authors and are not necessarily endorsed by the U.S. Army. 
Neck pain (NP) and low back pain (LBP) are two of the most common musculoskeletal conditions worldwide, particularly in occupations that involve manual labor, machine operations, and exposure to whole-body vibration (Cote et al., 2008; Hoy et al., 2012). Furthermore, female sex and older age have been shown to be associated with higher prevalence of NP/LBP (Cote et al., 2008; Hoy et al., 2012). The military is no exception and among various Military Occupation Specialties (MOS), military helicopter pilots and crew have the highest prevalence of NP/LBP (Gaydos, 2012; Salmon et al., 2011). However, due to a small proportion of female pilots and aircrew (4.4\% of all pilots) (Pena-Collazo, 2013), there have been few epidemiological studies to evaluate the prevalence of NP/LBP between female and male pilots and aircrew (Fraser et al., 2015; Nagai et al., 2014b; Simon-Arndt et al., 1997). Previously, based on the data from the Navy pilots and aircrew and their injury/medical diagnoses, there were no significant sex differences in back disorders (Simon-Arndt et al., 1997). Similarly, our preliminary study among the Army helicopter pilots and aircrew found the prevalence of NP/LBP to be similar between sexes (Nagai et al., 2014b). However, a recent report has revealed that female pilots were at a higher risk of neck pain related to flying (Fraser et al., 2015).

Previous research has identified several NP/LBP risk factors, such as longer total and night-vision goggle flight-hours, prolonged exposure to whole-body vibration, and poor sitting posture (Gaydos, 2012; Salmon et al., 2011). Due to confined cockpit space, height (taller pilots) appears to be a risk factor for LBP (Orsello et al., 2013). The sex-related difference in the prevalence of NP/LBP might be cancelled out due to the fact that males are generally taller than females, explaining the results from our preliminary epidemiological study (a lack of sex difference in NP/LBP) (Nagai et al., 2014b; Simon-Arndt et al., 1997). However, other than the difference in height, all pilots (regardless of sexes) must perform identical tasks as part of their occupation. In other words, female pilots (usually smaller stature) wear the same flight helmets/gear (same weight) and face the same occupation stress and associated risk factors, leading to a high prevalence of NP/LBP (Cote et al., 2008; Fraser et al., 2015; Hoy et al., 2012).

More recently, modifiable musculoskeletal characteristics have been investigated as possible risk factors of NP/LBP in Army helicopter pilots (Nagai et al., 2014a; Nagai et al., 2015a). Throughout this manuscript, the term "modifiable musculoskeletal characteristics" is operationally defined as musculoskeletal characteristics which can be changed through interventions. Muscular strength, muscular endurance, flexibility, biomechanics, and posture are generally considered as "modifiable musculoskeletal characteristics" though interventions. Those musculoskeletal characteristics are particularly important as they could be 
used to screen individuals and develop intervention programs to prevent future musculoskeletal injuries (Salmon et al., 2013). Pilots with a history of NP/LBP were found to have a significant reduction in cervical extension, cervical rotation, and lumbar lateral flexion range of motion (ROM) as well as trunk extension strength as compared to the pilots without a history of NP/LBP (Nagai et al., 2014a; Nagai et al., 2015a). It is speculated that female pilots, who reported NP related to flying in the recent report (Fraser et al., 2015), might have had reduced cervical musculoskeletal characteristics seen in pilots with a history of NP/LBP. As there were no sex differences in LBP (Simon-Arndt et al., 1997), there might not be any sex differences on trunk musculoskeletal characteristics. Literature coverage is lacking, with scarce available studies related to specific evaluation of sex differences in the cervical and trunk musculoskeletal characteristics among military helicopter pilots.

It has been reported that musculoskeletal and physiological characteristics are different between female and male Soldiers (Allison et al., 2015). In general, female Soldiers have been shown to have significantly less strength and aerobic/anaerobic capability, but better balance and greater flexibility compared to their male counterparts (Allison et al., 2015). Conflicting results have been reported for sex differences in the modifiable musculoskeletal characteristics related to NP/LBP in civilian population. While previous studies have reported that females have less cervical/trunk strength (Garces et al., 2002; Lee et al., 1999) and greater trunk ROM (Sullivan et al., 1994), other studies have reported no differences in cervical/trunk ROM (Dvorak et al., 1995; Trott et al., 1996). Mixed results might be due to the differences in methodologies used to collect those musculoskeletal characteristics and subjects used in the studies. In other words, previous civilian studies on various occupational workers (not including pilots) might not be relevant understanding NP/LBP as risk factors and working environments are very different from military helicopter pilots. Military pilots have spent years in physical, mental, and tactical training throughout their career; therefore, it can be argued that sex differences in modifiable musculoskeletal characteristics in civilian population might not be generalizable to military pilots. Therefore, the current preliminary study is essential to add knowledge to the literature.

Given a higher proportion of NP/LBP prevalence in the civilian females as well as sex differences in musculoskeletal characteristics, female military helicopter pilots may possess musculoskeletal characteristics that predispose them to a higher risk of NP/LBP. The purpose of the study was to compare previously established musculoskeletal risk factors of NP/LBP between sexes in military helicopter pilots. It was hypothesized that female pilots would exhibit less cervical and trunk strength and greater ROM than their male counterparts. Since few studies 
have examined cervical and trunk musculoskeletal characteristics in female military pilots, the results from the current investigation could be used to establish normative data as well as determine if sex differences exist in this population. If sex differences are found, then sex-specific preventative interventions could be developed and their effectiveness evaluated in reducing the prevalence of NP/LBP, which would improve force readiness.

\section{Method}

This study was a matched case-control design. Human subject approval was obtained from the Eisenhower Army Medical Center and the University of Pittsburgh Institutional Review Board. This study was a part of larger injury prevention and human performance optimization research initiatives and evaluated over 150 military helicopter pilots from the 101st Airborne Division (Air Assault). However, there were only 8 female pilots. A sample size was calculated based on the previous trunk rotation strength values with a large effect size and yielding at least 16 subjects to achieve the power of 0.80 and the $\alpha$ of 0.05 (Allison et al., 2015). Due to a small number of female pilots available, matching was used to adjust for the aircraft type, age ( \pm three years), and flight experience ( \pm two years). Total flight-hours vary within the group (minimum: 128 hours; maximum: 1900 hours). There were four aircraft types (4 UH-60 Black Hawk; 2 CH-47 Chinook; 1 AH-64 Apache, and 1 OH-58 Kiowa). Seat structures as well as vibration characteristics are different between the aircraft. In order to participate in the study, pilots had to be on a flight status and medically cleared for physical training. Subjects reported to the Warrior Human Performance Laboratory, and written informed consent was obtained from each pilot. Self-reported flight characteristics (flight experience and total flight-hours) and physical fitness information (most recent Army Physical Fitness Test [APFT] score) were collected. Height and mass were measured. All subjects were wearing the physical training uniform ( $t$-shirt and shorts) and a pair of running/workout shoes. Laboratory testing consisted of cervical and trunk strength and ROM assessments. Strength and ROM assessments were obtained in both directions (right and left), where applicable. The testing order was randomized. The reliability of the strength and ROM procedures has been established previously (Nagai et al., 2014a; Nagai et al., 2015a).

\section{Instruments}

Height and mass were measured using a standard stadiometer and scale (Seca North America, East Hanover, MD). Isometric cervical muscle strength (flexion, extension, right/left lateral flexion, and right/left rotation) was measured using a Lafayette handheld dynamometer (HHD) (Lafayette Instruments, Lafayette, 
IN). The Biodex Multi-Joint System 3 Pro (Biodex Medical Systems, Inc, Shirley, NY) was used to assess isokinetic trunk flexion, extension, and right/left rotation strength. Cervical flexion, extension, right/left lateral flexion, and right/left rotation active ROM were measured using the CROM 3 (Performance Attainment Associates, Lindstrom, MN). Two digital inclinometers (The Saunders Group Inc, Chaska, MN) were used to assess lumbar flexion, extension, right/left lateral flexion, and right/left rotation ROM.

\section{Procedures}

For the isometric cervical strength testing (flexion, lateral flexion, and rotation), the subject was in the supine position. For flexion, the HHD was positioned on the subject's forehead. For lateral flexion, the HHD was positioned above the ear and second researcher stabilized the opposite shoulder. For rotation, the HHD was positioned over the temporal line of the frontal bone. For extension, the subject was in the prone position. The HHD was positioned over the occiput. For all cervical strength testing, the same procedures were used. The warm-up set consisted of two trials at 50\% self-perceived maximum effort followed by two trials at $100 \%$ maximum effort. After a 60 -second rest, three maximal effort (100\%) trials were collected, with a 60-second rest between trials. The average of three trials for each strength assessment was normalized to body weight (\% BW) and used for analyses.

For trunk flexion, extension, and rotation strength measurements, the subject was positioned and stabilized according to the manufacturer's guidelines to ensure proper alignment for testing and to restrict accessory movements. The same procedures were used to both trunk flexion/extension and right/left rotation assessment. Three practice trials at $50 \%$ self-perceived maximum effort were performed to ensure proper movement, warm-up, and comfort throughout the available motion. Following a rest period of 60 seconds, each subject performed five repetitions of reciprocal concentric isokinetic trunk extension/flexion or right/left trunk rotation at $60 \% \mathrm{sec}$. The average peak torque of five trials in each direction was normalized to body weight $(\% \mathrm{BW})$ and used for analyses.

For cervical active ROM, the subject was seated on a wooden chair and fitted with the CROM according to the manufacturer's instructions. For cervical rotation ROM, the laser was project to the wall at the subject's eye level to ensure movement occurred in the correct plane of motion. The subject was instructed to move the head as far as possible until an uncomfortable stretch or pressure was felt. This position was held and the measurement recorded. Three practice trials were 
followed by three measured trials. The average of three trials in each direction was used for analyses.

For lumbar spine flexion ROM, the subject was seated and asked to actively forward flex, trying to reach the knees with the nose. For lumbar spine extension ROM, the subject was prone and asked to push down on the table with the hands and actively arch the trunk into extension while maintaining contact of the anterior superior iliac spines with the table. For lumbar spine lateral flexion, the subject was standing and asked to actively slide the hand down the lateral aspect of the ipsilateral thigh without pelvic rotation or trunk flexion, while keeping the knees straight and the feet in full contact with the ground. For lumbar spine rotation ROM, the subject was in a stooped position (trunk flexed to $90^{\circ}$ ), with the arms across the chest, feet in full contact with the floor, and knees straight. The subject was asked to rotate the trunk to the right or the left, with right side rotation operationally defined as rotating the right shoulder up. For each lumbar ROM testing, inclinometers were placed on T12 and L5 spinous processes, and the difference between those two inclinometers was recorded. The average of three trials in each direction was used for analyses.

\section{Statistical Analyses}

All statistical analyses were performed using IBM SPSS Statistics (version 20.0; IBM Corporation, Armonk, NY) and G*Power statistical software (version 3.1; The G*Power Team, Dusseldorf, Germany). Descriptive statistics (means and standard deviations) were calculated for each variable. Dependent variables were assessed for normality (Shapiro-Wilk test) by sex. Either paired t-tests (normally distributed) or Wilcoxon Signed Rank tests (not normally distributed) were performed. Significance was set at $p<0.05$. Effect size and power $(1-\beta)$ were calculated for each variable.

\section{Results}

Means and standard deviations for demographics, cervical and trunk strength, and ROM are presented in Tables I, II, and III, respectively. Female pilots were significantly shorter in height (Females: $166.1 \pm 7.7 \mathrm{~cm}$; Males: $175.0 \pm$ $6.8 \mathrm{~cm} ; \mathrm{p}=0.004$ ) and lighter in mass (Females: $67.9 \pm 10.6 \mathrm{~kg}$; Males: $79.5 \pm 5.8 \mathrm{~kg}$; $p=0.025)$. There were no sex differences in age, APFT score, flight experience, and total flight-hour $(p>0.05)$.

Female pilots had significantly lower cervical flexion strength (Females: $11.5 \pm 4.3 \% \mathrm{BW}$; Males: $18.9 \pm 4.2 \% \mathrm{BW} ; p=0.002)$, trunk flexion strength 
(Females: $226.6 \pm 32.9 \% \mathrm{BW}$; Males: $277.3 \pm 56.7 \% \mathrm{BW} ; p=0.040$ ), and trunk right rotation strength (Females: $114.9 \pm 23.9 \% \mathrm{BW}$; Males: $153.0 \pm 23.3 \% \mathrm{BW}$; $\mathrm{p}$ $=0.039)$ than male pilots. The other strength measures were not statistically significant between sexes $(p>0.05)$.

For flexibility measures, female pilots had significantly greater cervical right rotation (Females: $79.5 \pm 6.6^{\circ}$; Males: $73.7 \pm 5.0^{\circ} ; p=0.008$ ) and left rotation (Females: $78.5 \pm 5.0^{\circ}$; Males: $73.3 \pm 5.1^{\circ} ; p=0.019$ ). Other cervical flexibility measures were not statistically significant between sexes $(p>0.05)$. Additionally, there were no significant differences in lumbar spine ROM between sexes $(p>$ 0.05). Summaries of findings are displayed in Tables 1,2 , and 3.

Table 1

Means and SD for demographics, fitness, and flight characteristics

\begin{tabular}{lccccc}
\hline \multicolumn{1}{c}{ Dependent Variables } & Females & Males & $p$-value & Effect Size & Power \\
\hline Demographics & & & & & \\
Age (yrs)* & $27.6 \pm 4.2$ & $27.8 \pm 4.2$ & 0.317 & 0.048 & 0.051 \\
Height (cm) & $166.1 \pm 7.7$ & $175.0 \pm 6.8$ & 0.004 & 1.225 & 0.626 \\
Mass (kg) & $67.9 \pm 10.6$ & $79.5 \pm 5.8$ & 0.025 & 1.358 & 0.714 \\
APFT Score (points) & $277.6 \pm 27.6$ & $268.3 \pm 28.3$ & 0.505 & 0.333 & 0.095 \\
Flight Experience (yrs) & $4.8 \pm 4.1$ & $4.1 \pm 3.3$ & 0.451 & 0.188 & 0.064 \\
Total Flight-Hour (hr) & $851.6 \pm 709.2$ & $747.0 \pm 1001.8$ & 0.747 & 0.121 & 0.056 \\
\hline
\end{tabular}

Note: *Wilcoxon Signed Rank test; APFT = Army Physical Fitness Test

Table 2

Means and SD for strength

\begin{tabular}{lccccc}
\hline \multicolumn{1}{c}{ Dependent Variables } & Females & Males & $p$-value & Effect Size & Power \\
\hline Cervical Strength $(\% \mathrm{BW})$ & & & & & \\
Flexion & $11.5 \pm 4.3$ & $18.9 \pm 4.2$ & 0.002 & 1.741 & 0.899 \\
Extension & $24.9 \pm 3.7$ & $32.1 \pm 6.9$ & 0.062 & 1.301 & 0.677 \\
R Lateral Flexion* & $23.1 \pm 2.8$ & $25.3 \pm 4.1$ & 0.396 & 0.627 & 0.215 \\
L Lateral Flexion & $23.0 \pm 3.0$ & $26.6 \pm 4.9$ & 0.144 & 0.886 & 0.379 \\
R Rotation & $20.0 \pm 4.5$ & $20.4 \pm 2.8$ & 0.880 & 0.107 & 0.055 \\
L Rotation & $20.5 \pm 3.4$ & $21.1 \pm 2.8$ & 0.752 & 0.193 & 0.065 \\
Trunk Strength (\%BW) & & & & & \\
Flexion & $226.6 \pm 32.9$ & $277.3 \pm 56.7$ & 0.040 & 1.094 & 0.531 \\
Extension & $312.7 \pm 51.3$ & $364.7 \pm 78.4$ & 0.168 & 0.785 & 0.310 \\
R Rotation & $114.9 \pm 23.9$ & $153.0 \pm 23.3$ & 0.039 & 1.614 & 0.851 \\
L Rotation & $118.5 \pm 20.0$ & $142.2 \pm 31.0$ & 0.152 & 0.909 & 0.395 \\
\hline
\end{tabular}

Note: *Wilcoxon Signed Rank test; \%BW = percent of body weight; $\mathrm{R}=$ right; $\mathrm{L}=$ left. 
Nagai et al.: Sex Differences in Helicopter Pilots

Table 3

Means and SD for flexibility

\begin{tabular}{llllll}
\hline \multicolumn{1}{c}{ Dependent Variables } & Females & Males & $p$-value & Effect Size & Power \\
\hline Cervical ROM $\left(^{\circ}\right)$ & & & & & \\
$\quad$ Flexion & $60.2 \pm 8.4$ & $60.3 \pm 10.2$ & 0.985 & 0.011 & 0.050 \\
Extension & $75.4 \pm 9.3$ & $72.4 \pm 6.6$ & 0.426 & 0.372 & 0.107 \\
R Lateral Flexion & $53.5 \pm 5.1$ & $53.8 \pm 7.8$ & 0.917 & 0.046 & 0.051 \\
L Lateral Flexion & $54.5 \pm 7.3$ & $54.6 \pm 6.7$ & 0.986 & 0.014 & 0.050 \\
R Rotation & $79.5 \pm 6.6$ & $73.7 \pm 5.0$ & 0.008 & 0.991 & 0.454 \\
L Rotation & $78.5 \pm 5.0$ & $73.3 \pm 5.1$ & 0.019 & 1.030 & 0.483 \\
Lumbar Spine ROM $\left(^{\circ}\right)$ & & & & & \\
Flexion & $16.2 \pm 5.4$ & $16.1 \pm 5.0$ & 0.960 & 0.019 & 0.050 \\
Extension & $51.0 \pm 7.8$ & $46.4 \pm 10.2$ & 0.434 & 0.507 & 0.157 \\
R Lateral Flexion* & $26.1 \pm 5.5$ & $22.6 \pm 5.8$ & 0.263 & 0.619 & 0.211 \\
L Lateral Flexion & $26.4 \pm 5.1$ & $23.9 \pm 4.5$ & 0.270 & 0.520 & 0.163 \\
R Rotation & $10.8 \pm 4.3$ & $10.4 \pm 2.7$ & 0.891 & 0.111 & 0.055 \\
L Rotation* & $10.8 \pm 4.7$ & $10.6 \pm 2.5$ & 0.888 & 0.053 & 0.051 \\
\hline L & “Wil &
\end{tabular}

Note: $*$ Wilcoxon Signed Rank test; $\mathrm{ROM}=$ range of motion; $\mathrm{R}=$ right; $\mathrm{L}=$ left.

\section{Discussion}

Both NP and LBP are common musculoskeletal conditions in both civilian workers and military Soldiers, with NP/LBP being the most prevalent in military helicopter pilots and crew (Cote et al., 2008; Gaydos, 2012; Hoy et al., 2012; Salmon et al., 2011). While it is known that there is a higher prevalence of NP/LBP in civilian female workers (Cote et al., 2008; Hoy et al., 2012) and that sex differences in musculoskeletal characteristics related to NP/LBP exist (Allison et al., 2015; Garces et al., 2002; Lee et al., 1999; Sullivan et al., 1994; Tierney et al., 2005), sex-specific studies in military helicopter pilots are lacking, most likely due to the relatively low percentage of female pilots (Pena-Collazo, 2013). The current investigation evaluated sex differences in cervical and trunk musculoskeletal characteristics in Army helicopter pilots. Based on the current results, the hypotheses were partially supported as statistically significant differences were found between sexes for some, but not all, of the strength and ROM measures. More specifically, female pilots had significantly less cervical flexion strength, trunk flexion strength, and trunk right rotation strength; however, no sex differences were found for the remainder ( 7 out of 10) of the strength variables. Cervical right and left rotation ROM were the only flexibility variables that were significantly different between female and male pilots, with no significant differences in the majority (10 out of 12) of the ROM variables. 
In the current study, all strength values in male pilots were similar to the previous study with the same procedures, assuring the quality of the data/results collected in the present investigation (Nagai et al., 2014a). Cervical flexion strength was the only cervical strength measure that was significantly different between sexes. Previously, it was reported that civilian males demonstrated 30-40\% greater cervical flexion and extension strength than civilian females (Garces et al., 2002). This is in partial alignment with the findings of the current study as male military helicopter pilots had 39\% greater cervical flexion strength than female pilots; however, there was no statistically significant difference in cervical extension strength. A lack of significant sex differences in most of the cervical strength measures might be explained by musculoskeletal adaptations. It is speculated that cervical strength in female pilots gradually increased in response to occupational exposures. As military Soldiers, both female and male pilots engage in vigorous physical training daily, in addition flight-related physical demands. Although the frequent use of night vision goggles (NVG) has been identified as a potential risk factor of NP in military pilots (Harrison et al., 2015), it is possible that the added weight of NVG, over time, increases cervical strength in response to the additional mass. It seems that the additional mass is sufficient to induce changes in female pilots, but too low to induce changes in male pilots. Future investigations should evaluate those military pilots longitudinally to test those hypotheses.

The trunk strength values in the current study were similar to those previously reported using the same procedures (Nagai et al., 2015a). Trunk flexion strength and trunk right rotation strength were significantly different between sexes. The current findings were in accordance with previous studies reporting less trunk flexion and rotation strength in females (Allison et al., 2015; Shirado et al., 1995). A significant sex difference in trunk flexion, not trunk extension, was a surprising finding. Weaker trunk extension strength and trunk extension/flexion strength ratio are shown to be a risk factor for LBP in a civilian population (Lee et al., 1999). In military helicopter pilots, those characteristics have been associated with male military pilots with a history of LBP (Nagai et al., 2015a). One potential reason for a lack of sex difference on trunk extension strength is that pilots might have been training with resistance exercise. Compare to the Infantrymen, pilots usually perform their physical training in a small group. It means that it is easier for them to access to a gym and equipment. Deadlift and squat are common resistance exercises, and both exercises involve the trunk extensors as well as other lower extremity extensors. Additionally, they frequently perform 2-mile run, push-ups, and sit-ups as these are a part of the Army Physical Fitness Test (Army, 2012). Based on the current finding, female pilots should continue engaging resistance training, particularly on the abdominal muscles. A continued research on physical training on those modifiable musculoskeletal characteristics is warranted. 
Trunk right, not left, rotation strength was significant in the current study. Male pilots in the current study scored greater trunk right rotation strength (153.0\% BW) compared to the previous study (143.6\% BW) while trunk left rotation strength was similar in both studies (current: $142.2 \% \mathrm{BW}$ and previous: 141.0\%BW) (Nagai et al., 2015a). In other words, the current pilots exhibit a greater side-to-side difference. From the statistical point, trunk left strength had a large effect size (0.909) and less power (0.395), suggesting a small sample size. It would be interesting to see if both right and left rotation strength might become significant with a larger sample size.

For cervical ROM, the current values were similar to those reported previously using similar methods (Nagai et al., 2014a). Female pilots had significantly greater right and left cervical rotation ROM; however, there were no significant differences in the other cervical ROMs. This is in partial alignment with previous studies, which reported greater cervical ROM in all directions in female civilians as compared to their male counterparts (Ferrario et al., 2002). While it is unclear why female pilots had significantly increased cervical rotation ROM, but not flexion/extension and lateral flexion ROM, it might be beneficial from a tactical and medical perspective to have greater cervical ROM. Tactically, when flying with $\mathrm{NVG}$, a field of view is reduced to 40 degrees in the vertical and horizontal directions (Harrison et al., 2015); therefore, greater cervical rotation motion will be required to scan the entire area in order to decrease the likelihood of missing a structure or target. Medically, significant reductions in neck extension and rotation were identified as potential musculoskeletal risk factors for NP (Ang, 2008; Nagai et al., 2014a; Van den Oord et al., 2010). Based on the results from the current study and previously identified NP risk factors, engaging in cervical rotation ROM exercise might be important for male pilots to reduce the risk of developing NP.

Lastly, there were no sex differences in lumbar spine ROM. The current values for lumbar spine ROM were similar to those in previous studies (Keeley et al., 1986; Mellin et al., 1991; Nagai et al., 2015a). Although not significant, female pilots were more flexible in lumbar spine extension and right/left lateral flexion ROM than male pilots $\left(4.6^{\circ}, 3.5^{\circ}\right.$, and $2.5^{\circ}$, respectively). Male pilots should be aware that a lack of lumbar spine lateral flexion ROM was associated with pilots with a history of LBP (Nagai et al., 2015a). Further, it is speculated that prolonged sitting in a confined cockpit gradually diminishes lumbar spine ROM and increase the thoracic spine and shoulder roundedness, known as "halo hunch" (Gaydos, 2012). In turn, this awkward sitting posture is thought to make the matter worse for helicopter pilots over their career (Gaydos, 2012). To support this contention, we have analyzed a group of pilots based on their total flight-hours and found 
significant reductions in lumbar spine extension and lateral flexion ROM in the pilots with over 2000 total flight-hours when compared with the pilots with less than 1000 total flight-hours (Nagai et al., 2015b). Those results suggest a routine stretching program for all helicopter pilots, especially male pilots over 2000 total flight-hours.

There are limitations. Since the current study was a preliminary study to compare cervical and trunk musculoskeletal characteristics between female and male pilots, the sample size was small, increasing the chance of a type II error. Among the dependent variables with the significance, only two (out of seven variables) reached the power of 0.80 . Therefore, the results should be used as preliminary findings and interpreted cautiously. Future study utilizing a larger sample size is warranted. The current study only examined sex differences in cervical and lumbar strength and ROM. As the number of female pilots grow, the effects of other risk factors (e.g., age, total-flight-hours, and NVG hours) as well as the interaction among these factors and between sexes should be examined. Also, the current study examined muscular strength rather than muscular endurance or fatigue, which may be more important to examine relative NP/LBP.

\section{Conclusion}

There are some sex differences in cervical and trunk strength and ROM in pilots in the current study; however, many of the differences in cervical and trunk strength and ROM, reported in a civilian population, are not present in military helicopter pilots. This indicates that there is a unique cervical and lumbar musculoskeletal profile in pilots, particularly relative to sex. Given that cervical and trunk musculoskeletal characteristics are important relative to injury risk and tactical performance, continue efforts are warranted for three main areas to address. First, other modifiable musculoskeletal characteristics (i.e. muscular endurance, hand-eye coordination, and postural control) should be explored. As stated before, modifiable musculoskeletal characteristics are essential as they could guide us developing evidence-based intervention programs (Salmon et al., 2013). In fact, previous intervention studies have demonstrated a reduction in neck pain and musculoskeletal injuries (Ang et al., 2009; Sell et al., 2016). Second, since both female and male pilots must perform the identical tasks, they should be trained to a level that would reduce NP/LBP. This could mean more emphasis on flexibility for male pilots and strength for female pilots. Lately, sex differences in the cervical and trunk musculoskeletal characteristics of military helicopter pilots do not appear to parallel those of a civilian population, requiring a larger sample size to build a database of musculoskeletal characteristics (NP/LBP risk factors) in this population. 


\section{References}

Allison, K. F., Keenan, K. A., Sell, T. C., Abt, J. P., Nagai, T., Deluzio, J., McGrail, M., \& Lephart, S. M. (2015). Musculoskeletal, biomechanical, and physiological gender differences in the US Military. Army Medical Department Journal, Apr-Jun, 22-32.

Ang, B. O. (2008). Impaired neck motor function and pronounced pain-related fear in helicopter pilots with neck pain - a clinical approach. $J$ Electromyogr Kinesiol, 18(4), 538-549.

Ang, B. O., Monnier, A., \& Harms-Ringdahl, K. (2009). Neck/shoulder exercise for neck pain in air force helicopter pilots: a randomized controlled trial. Spine (Phila Pa 1976), 34(16), E544-551.

Army (2012). Army Physical Readiness Training. Field Manual 7-22. Washington, DC: Headquarters, Department of the Army.

Cote, P., van der Velde, G., Cassidy, J. D., Carroll, L. J., Hogg-Johnson, S., Holm, L. W., Carragee, E. J., Haldeman, S., Nordin, M., Hurwitz, E. L., Guzman, J., Peloso, P. M., Bone, Joint Decade - Task Force on Neck, P., $\&$ Its Associated, D. (2008). The burden and determinants of neck pain in workers: results of the Bone and Joint Decade 2000-2010 Task Force on Neck Pain and Its Associated Disorders. Spine (Phila Pa 1976), 33(4 Suppl), S60-74. doi:10.1097/BRS.0b013e3181643ee4

Dvorak, J., Vajda, E. G., Grob, D., \& Panjabi, M. M. (1995). Normal motion of the lumbar spine as related to age and gender. European Spine Journal, $4(1), 18-23$.

Ferrario, V. F., Sforza, C., Serrao, G., Grassi, G., \& Mossi, E. (2002). Active range of motion of the head and cervical spine: a three-dimensional investigation in healthy young adults. Journal of Orthopedic Research, 20(1), 122-129. doi:10.1016/S0736-0266(01)00079-1

Fraser, W. D., Crowley, J. S., Shender, B. S., \& Lee, V. M. (2015). Multinational survey of neck pain in rotor-wing aircrew, The Technical Cooperation Program Technical Report DOC-HUM-01-2015 
Garces, G. L., Medina, D., Milutinovic, L., Garavote, P., \& Guerado, E. (2002). Normative database of isometric cervical strength in a healthy population. Medical Science of Sports and Exercise, 34(3), 464-470.

Gaydos, S. J. (2012). Low back pain: considerations for rotary-wing aircrew. Aviation, Space, and Environmental Medicine, 83(9), 879-889.

Harrison, M. F., Coffey, B., Albert, W. J., \& Fischer, S. L. (2015). Night vision goggle-induced neck pain in military helicopter aircrew: a literature review. Aerospace Medicine and Human Performance, 86(1), 46-55. doi:10.3357/AMHP.4027.2015

Hoy, D., Bain, C., Williams, G., March, L., Brooks, P., Blyth, F., Woolf, A., Vos, T., \& Buchbinder, R. (2012). A systematic review of the global prevalence of low back pain. Arthritis \& Rheumatism, 64(6), 2028-2037. doi:10.1002/art.34347

Keeley, J., Mayer, T. G., Cox, R., Gatchel, R. J., Smith, J., \& Mooney, V. (1986). Quantification of lumbar function. Part 5: Reliability of range-of-motion measures in the sagittal plane and an in vivo torso rotation measurement technique. Spine (Phila Pa 1976), 11(1), 31-35.

Lee, J. H., Hoshino, Y., Nakamura, K., Kariya, Y., Saita, K., \& Ito, K. (1999). Trunk muscle weakness as a risk factor for low back pain. A 5-year prospective study. Spine, 24(1), 54-57.

Mellin, G., Kiiski, R., \& Weckstrom, A. (1991). Effects of subject position on measurements of flexion, extension, and lateral flexion of the spine. Spine (Phila Pa 1976), 16(9), 1108-1110.

Nagai, T., Abt, J. P., Sell, T. C., Clark, N. C., Smalley, B. W., Wirt, M. D., \& Lephart, S. M. (2014a). Neck proprioception, strength, flexibility, and posture in pilots with and without neck pain history. Aviation, Space, and Environmental Medicine, 85(5), 529-535.

Nagai, T., Abt, J. P., Sell, T. C., Keenan, K. A., Clark, N. C., Smalley, B. W., Wirt, M. D., \& Lephart, S. M. (2015a). Lumbar spine and hip flexibility and trunk strength in helicopter pilots with and without low back pain history. Work, 52(3), 715-722. doi:10.3233/WOR-152192 
Nagai, T., Abt, J. P., Sell, T. C., Lovalekar, M., Smalley, B. W., Wirt, M. D., \& Lephart, S. M. (2015b). Exposure-related differences on neck and trunk musculoskeletal characteristics in US Army helicopter pilots. Aerospace Medical Association Annual Meeting.

Nagai, T., Lovalekar, M., Allison, K. F., Abt, J. P., Sell, T. C., Smalley, B. W., Wirt, M. D., \& Lephart, S. M. (2014b). Prevalence of neck pain and low back pain in a Combat Aviation Brigade. American College of Sports Medicine.

Orsello, C. A., Phillips, A. S., \& Rice, G. M. (2013). Height and in-flight low back pain association among military helicopter pilots. Aviation, Space, and Environmental Medicine, 84(1), 32-37.

Pena-Collazo, S. (2013). Women in Combat Arms: A Study in the Global War on Terror. (Thesis), U.S. Army Command and General Staff College, Fort Leavenworth, KS.

Salmon, D. M., Harrison, M. F., \& Neary, J. P. (2011). Neck pain in military helicopter aircrew and the role of exercise therapy. Aviation, Space, and Environmental Medicine, 82(10), 978-987.

Salmon, D. M., Harrison, M. F., Sharpe, D., Candow, D., Albert, W. J., \& Neary, J. P. (2013). Exercise therapy for improved neck muscle function in helicopter aircrew. Aviation, Space, and Environmental Medicine, 84(10), 1046-1054.

Sell, T. C., Abt, J. P., Nagai, T., Deluzio, J. B., Lovalekar, M., Wirt, M. D., \& Lephart, S. M. (2016). The Eagle Tactical Athlete Program Reduces Musculoskeletal Injuries in the 101st Airborne Division (Air Assault). Military Medicine, 181(3), 250-257. doi:10.7205/MILMED-D-14-00674

Shirado, O., Ito, T., Kaneda, K., \& Strax, T. E. (1995). Concentric and eccentric strength of trunk muscles: influence of test postures on strength and characteristics of patients with chronic low-back pain. Archives of Physical Medicine and Rehabilitation, 76(7), 604-611.

Simon-Arndt, C. M., Yuan, H., \& Hourani, L. L. (1997). Aircraft type and diagnosed back disorders in U.S. Navy pilots and aircrew. Aviation, Space, and Environmental Medicine, 68(11), 1012-1018. 
Sullivan, M. S., Dickinson, C. E., \& Troup, J. D. (1994). The influence of age and gender on lumbar spine sagittal plane range of motion. A study of 1126 healthy subjects. Spine (Phila Pa 1976), 19(6), 682-686.

Tierney, R. T., Sitler, M. R., Swanik, C. B., Swanik, K. A., Higgins, M., \& Torg, J. (2005). Gender differences in head-neck segment dynamic stabilization during head acceleration. Medicine \& Science in Sports \& Exercise, 37(2), 272-279.

Trott, P. H., Pearcy, M. J., Ruston, S. A., Fulton, I., \& Brien, C. (1996). Threedimensional analysis of active cervical motion: the effect of age and gender. Clinical Biomechanics (Bristol, Avon), 11(4), 201-206.

Van den Oord, M. H., De Loose, V., Sluiter, J. K., \& Frings-Dresen, M. H. (2010). Neck strength, position sense, and motion in military helicopter crew with and without neck pain. Aviation, Space, and Environmental Medicine, 81(1), 46-51. 